\title{
Qualidade nutricional de barras de chocolate adicionadas de castanhas de baru ${ }^{1}$
}

Nutritional quality of chocolate bars with baru nuts

Cristina Correa da Silva Lubas ${ }^{2}$

Camila Jordão Cândido ${ }^{3}$

Suene Vanessa da Silva Souza ${ }^{4}$

Rita de Cássia Avellaneda Guimarães ${ }^{5}$

${ }^{1}$ Agradecemos ao Conselho Nacional de Desenvolvimento Científico e Tecnológico (CNPq) pelo apoio financeiro ao presente trabalho.

${ }^{2}$ Acadêmica do Curso de Nutrição, Universidade Federal de Mato Grosso do Sul (UFMS). E-mail: cristinacorreas_@hotmail.com

${ }^{3}$ Doutoranda da Pós-Graduação em Saúde e Desenvolvimento na Região Centro-Oeste, Universidade Federal de Mato Grosso do Sul (UFMS). E-mail: cahjordao@gmail.com

${ }^{4}$ Acadêmica do Curso de Nutrição, UFMS. E-mail: suene.vs@gmail.com

${ }^{5}$ Docente da Pós-Graduação em Saúde e Desenvolvimento na Região Centro-Oeste/Curso de Nutrição, UFMS. E-mail: rita.guimaraes@ufms.br 


\section{RESUMO ABSTRACT}

Chocolate é definido como produto obtido a partir da mistura de derivados de cacau com outros ingredientes, contendo no mínimo $25 \%$ de sólidos totais de cacau. A castanha de baru (Dipteryx alata Vog.) é amplamente encontrada do Cerrado brasileiro podendo ser apreciada na alimentação. Este trabalho teve por objetivo avaliar a qualidade do produto na questão microbiológica e composição centesimal do produto. Barras de chocolates acrescidas de castanhas de baru foram testadas em diferentes concentrações do fruto. As análises microbiológicas e composição centesimal foram realizadas. Os produtos foram analisados e considerados aptos para o consumo. 0 estudo da composição centesimal foi satisfatório, especialmente para carboidratos nas formulações contendo 35\% de amêndoa, assim como baixo teor de lipídios e calorias nas formulações contendo $15 \%$ de amêndoa. Os resultados obtidos foram muito satisfatórios, tendo em vista que as chances de contaminação aumentam quando o alimento é produzido artesanalmente.

PALAVRAS-CHAVE

$\begin{aligned} \text { baru } & \text { baru } \\ \text { chocolate } & \text { chocolate } \\ \text { análise microbiológica } & \text { microbiological analysis } \\ \text { composição centesimal } & \text { chemical composition }\end{aligned}$

baru baru chocolate chemical composition microbiological and chemical composition were carried out. produced by hand.

\section{KEY WORDS} obtained from the mixture of cocoa products with other ingredients, containing at least 25\% total cocoa solids. The baru nut (Dipteryx alata Vog.) Its widely found in the Brazilian Cerrado can be appreciated in food. This study aimed to evaluate the quality of the product in question composition of the product. chocolate bars plus baru nuts were tested at different concentrations of the fruit. The microbiological and chemical The products were analyzed and considered fit for consumption. The study of the chemical composition was satisfactory, especially for formulations containing carbohydrates in 35\% almond, as well as low in calories and lipids in formulations containing $15 \%$ almond. The results were very satisfactory, given that the chances of contamination increase when food is 


\section{INTRODUÇÃo}

De acordo com a Resolução RDC n. 264, de 22 de setembro de 2005, chocolate é definido como produto obtido a partir da mistura de derivados de cacau (massa, pasta ou liquor de cacau, cacau em pó e ou manteiga de cacau) com outros ingredientes, contendo no mínimo $25 \%$ (g/100 g) de sólidos totais de cacau (ANVISA, 2005).

Por ser o cacau o ingrediente principal do chocolate, as características e as operações envolvidas no seu processamento (colheita, fermentação e secagem), que iniciam na propriedade agrícola, seguido dos processos de moagem do cacau, conchagem e temperagem, influenciam muito na qualidade final do chocolate (LEITE et al. 2013).

Outra matéria prima empregada na formulação dos chocolates do presente trabalho é a castanha do baru. A castanha é proveniente do fruto do barueiro (Dipteryx alata Vog.) da família Leguminosae, uma espécie arbórea bastante produtiva que ocorre na região do Cerrado brasileiro. Por todo o território brasileiro, é conhecido por diversos nomes, como barujó, baruí, baruzeiro, cumbaru, coco-feijão, cumaru e pau cumaru (VERA; SOUZA, 2009).

Existem poucos estudos que demonstram as características da composição de sua semente. 0 baru necessita de estudos mais aprofundados para ser mais bem aproveitado tanto pela indústria alimentícia quanto pela indústria farmacêutica (GUIMARÃES, 2009).

Para a produção segura de um alimento, é necessário o estabelecimento de normas, limites e padrões. É importante exercer inspeção, controle, fiscalização e vigilância para que contaminantes que causam perigo à saúde estejam ausentes ou abaixo do limite de risco (SOUSA et al., 2010).

Os coliformes termotolerantes (Coliformes a $45^{\circ} \mathrm{C}$ ), bolores e leveduras são utilizados como indicadores higiênico-sanitários. 
Tendo em vista que o habitat natural dos coliformes é o trato intestinal, a presença desses microrganismos em alimentos demonstra contaminação de origem fecal direta ou indireta, podendo ser por contato direto com fezes contaminadas ou por falta de assepsia do manipulador. As bactérias do gênero Salmonella são os microrganismos envolvidos mais frequentemente nos surtos de doenças de origem alimentar nos seres humanos, incluindo vários tipos de alimentos, principalmente os de origem animal (TEJADA et al., 2012).

É preciso extremo cuidado com a contaminação cruzada de alimentos, que deve ser evitada. Carnes cruas devem ser mantidas separadas dos alimentos cozidos e alimentos prontos para o consumo. Mãos, tábuas de corte, contadores mecânicos e manuais, facas e outros utensílios devem ser lavados e higienizados após tocar em alimentos crus. As mãos devem ser sempre lavadas antes de manipular alimentos e entre a manipulação de diferentes itens alimentares (CENTERS OF DESEASE CONTROL AND PREVENTION, 2015).

Entre as espécies do gênero Bacillus, uma das mais importantes na indústria de alimentos é o $B$. cereus. Este possui capacidade de produzir toxinas, responsáveis por toxinfecções alimentares, enzimas extracelulares, que determinam o potencial de deterioração, e esporos. Sabe-se que a forma esporulada é resistente a processos de cocção (ROBINSON; PHILL, 1987).

Mediante a possibilidade de episódios de toxinfecções alimentares provocadas pelo consumo de chocolate, justifica-se no presente estudo verificar as condições higiênico-sanitárias no momento da elaboração de chocolates em barra desse alimento e avaliar a composição centesimal (TEJADA et al., 2012). 


\section{MATERIAL E MÉTODOS}

\subsection{Análise microbiológica}

Os chocolates em barra classificados como "amargo" e "meio-amargo" foram adquiridos comercialmente na cidade de Campo Grande, MS, assim como frutos de baru, que foram coletados em diferentes pontos da cidade no período de agosto a novembro de 2014. Os frutos foram quebrados em despolpador mecânico, e a castanha utilizada na elaboração do chocolate.

As castanhas de baru foram torradas até atingir a temperatura de $160^{\circ} \mathrm{C}$ por 40 minutos em forno convencional. Após esfriarem em temperatura ambiente, as películas da parte externa das castanhas foram removidas manualmente. Em seguida, foram trituradas em liquidificador doméstico e acrescentadas em chocolate derretido.

Barras de chocolates acrescidas de castanhas de baru foram testadas nas concentrações do fruto a 15\%, 25\% e 35\% com chocolate "amargo" e "meio-amargo," para melhor avaliar as características finais sensoriais do produto final.

As análises microbiológicas foram realizadas para Bacillus cereus, Salmonella sp, bolores, leveduras, e Coliformes a $45^{\circ} \mathrm{C}$, de acordo com a Resolução-RDC n. 12, de 2 de janeiro de 2001, da Agência Nacional de Vigilância Sanitária (ANVISA) do Ministério da Saúde (BRASIL, 2001), para farinhas, massas alimentícias, doces e produtos de panificação (industrializados e embalados) e similares, em barra ou em outras formas, com ou sem adições.

A regulamentação técnica da ANVISA utilizada foi Categoria 5 - Balas, confeitos, bombons, chocolates e similares. As amostras foram analisadas em repetição triplicata.

A metodologia utilizada foi da American Public Health Association, descrita no CompendiumofMethodsfor the Microbiological Examination of Foods (VANDERZANT; SPLITTSTOESSER, 1992). Os 
testes microbiológicos foram realizados em laboratório de microbiologia de alimentos, sendo realizados em triplicata.

\subsection{Composição centesimal}

Na formulação de chocolate acrescido de castanhas de baru, foram testadas concentrações do fruto a 15\%, 25\% e 35\%, em barras de chocolate "amargo" e "meio amargo", para melhor avaliar as características finais do produto elaborado. As análises da composição centesimal foram realizadas em triplicata de acordo com as normas analíticas do Instituto Adolfo Lutz (BRASIL, 2005). A análise da umidade foi realizada em estufa a $105^{\circ} \mathrm{C}$ até peso constante. A análise do resíduo mineral fixo foi realizada por incineração em mufla a $550^{\circ} \mathrm{C}$. 0 teor de lipídeos totais foi determinado por extração com éter etílico em aparelho de Soxhlet.

A proteína foi determinada pelo conteúdo de nitrogênio total, segundo método do micro Kjedahl e o fator de 6,25 usado para a conversão do teor de nitrogênio em proteína bruta. Os carboidratos foram determinados pelo método de Lane-Eynon baseado na redução do cobre. 0 teor de fibra alimentar foi determinado por diferença total. 0 valor energético total proveniente dos nutrientes foi expresso em quilocalorias (kcal), estimada a partir dos fatores de conversão de Atwater: $\mathrm{kcal}=(4$ x g proteína $)$ + (4 x g carboidratos) + (9 x g lipídios) (MERRIL; WATT, 1973).

\subsection{Análise estatística}

As análises estatísticas foram realizadas em duplicata e os dados obtidos submetidos à análise de variância, e as médias serão comparadas entre si pelo teste Tukey, a 5\% de probabilidade. 


\section{RESULTADOS E DISCUSSÃO}

\subsection{Análise microbiológica}

De acordo com a Resolução-RDC n. 12, de 2 de janeiro de 2001 (ANVISA), para chocolate e produtos similares em barra ou na forma de bombom, adicionado ou não de outros ingredientes secos, devem ser analisados níveis de Coliformes a $45^{\circ}$, estafilococos coagulase positiva e Salmonella sp. Desse modo, os níveis tolerados são respectivamente: 10 UFC/g, $5 \times 10^{2}$ UFC/25g e ausência (BRASIL, 2001).

Amostras de chocolate "amargo" e "meio amargo" nas concentrações de castanhas de baru de 15\%, 25\% e 35\% foram analisadas e consideradas todas aptas para o consumo por estarem abaixo ou igual aos estabelecidos para o consumo. Não foi detectada a presença de bolores e leveduras.

Um estudo feito por Sousa et al. (2010) avaliou as condições higiênico-sanitárias de estabelecimentos produtores de bombons de chocolate recheados com frutas regionais, produzidos na cidade de Belém, PA. Nesse estudo, não foi observada a presença de Salmonella sp e Estafilococus coagulase positiva em nenhum dos bombons avaliados, mas foi detectada a presença de Coliformes a $45^{\circ} \mathrm{C}$ nos bombons de produção caseira. Foram identificadas bactérias aeróbias mesófilas e bolores e leveduras na produção industrial e caseira.

Em outro estudo realizado na cidade de Apucarana, PR, em 2011, foram analisadas as condições microbiológicas de bombons caseiros de fruta (morango) e do tipo crocante. 0 maior nível de contaminação microbiológica foi apresentado pelo bombom de fruta, porém ambos apresentaram resultados para bolores e leveduras e coliformes totais acima do padrão estabelecido pela legislação (REIS, 2011).

Tejada et al. (2012) verificaram a qualidade microbiológica de chocolates ao leite artesanais produzidos e comercializados na região sul do estado do Rio Grande do Sul. Nesse estudo, 32,5\% das 
amostras analisadas mostraram-se impróprias para o consumo. Dessas amostras, 27,5\% apontaram contagens acima dos limites estabelecidos para bolores e leveduras, sendo que destas 17,5\% mostraram apenas esse parâmetro fora dos padrões. Em relação a Coliformes totais, $12,5 \%$ estavam com valores acima do aceitável.

\subsection{Composição centesimal}

Os valores obtidos nas análises centesimais de $100 \mathrm{~g}$ de cada barra estão apresentados no Quadro 1.

\begin{tabular}{|c|c|c|c|}
\hline $\begin{array}{l}\text { Parâmetros* } \\
(\text { g.100 g-1) }\end{array}$ & $\begin{array}{c}15 \% \text { de } \\
\text { amêndoa de } \\
\text { baru }\end{array}$ & $\begin{array}{c}25 \% \text { de } \\
\text { amêndoa de } \\
\text { baru }\end{array}$ & $\begin{array}{c}35 \% \text { de } \\
\text { amêndoa de } \\
\text { baru }\end{array}$ \\
\hline \multicolumn{4}{|c|}{ Chocolate meio amargo } \\
\hline Umidade & $1,215 \pm 0,66^{a}$ & $1,302 \pm 0,022^{\mathrm{a}}$ & $1,771 \pm 0,007^{\mathrm{b}}$ \\
\hline Resíduo mineral fixo & $1,580 \pm 0,01^{\mathrm{a}}$ & $1,830 \pm 0,010^{\mathrm{a}}$ & $1,950 \pm 0,04^{b}$ \\
\hline Proteína & $7,430 \pm 0,16^{\mathrm{a}}$ & $9,072 \pm 0,590^{a}$ & $11,400 \pm 0,04^{\mathrm{b}}$ \\
\hline Lipídios & $26,890 \pm 0,02^{\mathrm{a}}$ & $31,978 \pm 0,360^{b}$ & $36,521 \pm 0,72^{b}$ \\
\hline Carboidratos & $62,885 \pm 0,50^{\mathrm{a}}$ & $55,822 \pm 0,980^{\mathrm{a}}$ & $48,364 \pm 0,77^{\mathrm{b}}$ \\
\hline Calorias (kcal) & $523,27 \mathrm{kcal}$ & $547,378 \mathrm{kcal}$ & $567,745 \mathrm{kcal}$ \\
\hline \multicolumn{4}{|c|}{ Chocolate amargo } \\
\hline Umidade & $1,687 \pm 0,175^{\mathrm{a}}$ & $1,678 \pm 0,123^{a}$ & $2,013 \pm 0,239^{b}$ \\
\hline Resíduo mineral fixo & $1,632 \pm 0,030^{a}$ & $1,759 \pm 0,021^{\mathrm{a}}$ & $1,868 \pm 0,018^{\mathrm{b}}$ \\
\hline Proteínas & $6,210 \pm 0,150^{a}$ & $9,639 \pm 0,306^{a}$ & $13,160 \pm 0,171^{b}$ \\
\hline Lipídios & $34,510 \pm 0,110^{\mathrm{a}}$ & $35,076 \pm 0,192^{a}$ & $36,217 \pm 1,072^{b}$ \\
\hline Carboidratos & $55,960 \pm 0,457^{\mathrm{a}}$ & $51,848 \pm 0,641^{a}$ & $46,745 \pm 0,986^{b}$ \\
\hline Calorias (kcal) & 559,27 & 561,632 & 565,573 \\
\hline
\end{tabular}

Quadro 1 - Composição centesimal de barras de chocolate amargo e meio amargo com diferentes concentrações de amêndoa de baru * Dados fornecidos com média e desvio-padrão.

** Dados apresentados como média \pm desvio-padrão. Letras iguais na mesma linha não diferem entre si $(\mathrm{p}>0.05)$.

Em relação ao conteúdo de umidade para o chocolate meio amargo, verificou-se o maior teor para a formulação contendo $35 \%$ de amêndoas de baru e, para o chocolate amargo, o maior teor 
encontrado também foi na formulação com 35\% de amêndoas. Para o resíduo mineral fixo, teores mais elevados foram encontrados nas formulações contendo 35\% de amêndoa de baru, tanto para o chocolate meio amargo quanto para o chocolate amargo. Santos et al. (2011), ao avaliarem biscoito de chocolate acrescido de polvilho, obtiveram valores de umidade de 2,10 $\pm 0,12$ g. $100^{-1}$ e resíduo mineral fixo de 1,42 $\pm 0,17$ g. $100^{-1}$, sendo este último valor inferior ao das formulações de chocolate contendo $35 \%$ de amêndoa de baru. Mosca et al. (2014) reportaram valores de umidade e resíduo mineral fixo para bolo de chocolate adicionado de grãos de feijão cozidos de, respectivamente, 35,45 $\pm 0,01$ g. $100 \mathrm{~g}^{-1}$ e 0,75 $\pm 0,03$ g. $100 \mathrm{~g}^{-1}$, sendo verificado teor de umidade superior quando comparado ao presente trabalho.

Quanto ao conteúdo de proteínas, notou-se o maior teor na formulação contendo 35\% de amêndoas de baru, para o chocolate meio amargo e amargo. Para lipídios, o menor teor avaliado foi para a formulação de 15\%, em ambos os tipos de chocolate utilizado. Reis (2011), ao avaliar bombons de morango elaborados artesanalmente, encontrou, no primeiro lote de produção, teores de proteína de 6,53 \pm 0,548 g. $100 \mathrm{~g}^{-1}$ e de lipídios de 14,01 $\pm 1,073$ g. $100 \mathrm{~g}^{-1}$, sendo o valor das proteínas inferior ao das formulações contendo 35\% de amêndoas de baru, assim como os de lipídios foi inferior ao das formulações contendo 15\%. Richter e Lannes (2007), ao formularem bombons para dietas especiais, obtiveram valores de 8,57 $\pm 0,010$ g. $100 \mathrm{~g}^{-1}$ para proteína, e 25,40 $\pm 0,009$ g. $100 \mathrm{~g}^{-1}$ para lipídios. 0 valor para proteínas foi inferior ao das formulações apresentadas pelo presente trabalho com 35\% de amêndoas de baru, enquanto que, o valor de lipídios foi aproximado ao das formulações de chocolate meio amargo acrescido de 15\% de amêndoas de baru, e inferior ao da formulação de chocolate amargo, com a mesma concentração de amêndoas.

Em relação aos carboidratos, observou-se menor teor na formulação contendo 35\% de amêndoas de baru em ambos os 
chocolates, meio amargo e amargo, e menor teor de calorias nas formulações contendo $15 \%$ das amêndoas, para os dois tipos de chocolate. Em uma formulação com adição de inulina em bolo de chocolate, Lotici et al. (2013) reportaram valores de carboidratos de $44,24 \pm 0,48$ g. $100 \mathrm{~g}^{-1}$ e $376,81 \pm 0,98 \mathrm{kcal}$, sendo o valor de carboidrato inferior ao da formulação contendo $35 \%$ de amêndoas, e o valor das calorias superior ao da formulação contendo 15\% de amêndoas de baru. Miquelim (2006) ao avaliar bombons recheados com preparo de maracujá, obteve para carboidratos o valor de 21,4 g. $100 \mathrm{~g}^{-1}$, e para calorias o valor de $607,44 \mathrm{kcal}$. Quando comparado ao presente estudo, o valor de carboidrato é inferior ao das formulações contendo 35\% de amêndoas de baru, e as calorias são superiores às das formulações contendo $15 \%$ de amêndoas.

\section{CONCLUSÃO}

Os chocolates utilizados para o presente estudo foram produzidos de modo artesanal, ainda sim se mostraram aptos para o consumo com níveis abaixo ou igual aos estabelecidos para amostra indicativa de acordo com a legislação vigente. Além disso, não foi detectada a presença de bolores e leveduras. É válido salientar que as castanhas de baru obtidas para a composição do chocolate também passaram por processo manual (coleta, despolpamento, torragem da castanha e trituramento) até serem incorporadas, de fato, às barras de chocolate analisadas.

Os resultados obtidos foram muito satisfatórios, tendo em vista que as chances de contaminação aumentam quando o alimento é produzido artesanalmente. Porém, quando alimentos são fabricados e destinados ao consumo humano, é necessário fiscalização de órgãos competentes, padronização de procedimentos de higiene e implementação de Boas Práticas de Fabricação. 0 estudo da composição centesimal apresentou formulações contendo amêndoas de baru que foram satisfatórias, apresentando alto 
teor de umidade, resíduo mineral fixo, e proteína, e baixo teor de carboidratos nas formulações contendo 35\% de amêndoa, assim como baixo teor de lipídios e calorias nas formulações contendo $15 \%$ de amêndoa. Uma porção de $25 \mathrm{~g}$ de cada barra fornece nutrientes, boa fonte de energia, e pode ser consumida rotineiramente. Além disso, valoriza a aplicação de frutos do Cerrado em elaboração de novos produtos, trazendo-lhes mais visibilidade. Todas as medidas que auxiliam na prevenção de contaminações devem ser colocadas em prática, tendo em vista que a ingestão de alimentos contaminados podem causar graves prejuízos a saúde, podendo levar a morte em casos mais graves.

\section{REFERÊNCIAS}

BRASIL. Ministério da Saúde. Resolução - RDC n. 12, de 02 de janeiro de 2001. Aprova o regulamento técnico sobre padrões microbiológicos para alimentos.

. Agência Nacional de Vigilância Sanitária. Resolução n. 264, de 22 de setembro de 2005. Regulamento técnico para chocolate e produtos do cacau. Diário Oficial da União. Poder Executivo, de 23 de setembro de 2005.

CENTERS OF DESEASE CONTROL AND PREVENTION (CDC). Salmonella. 2015. Disponível em: <http://www.cdc.gov/salmonella/general/ prevention.html>. Acesso em: 21 ago. 2015.

LEITE, P. B.; LANNES, S. C. S.; RODRIGUES, A. M.; SOARES, F. A. S. M.; SOARES, S. E.; BISPO, E. S. Rheological study of chocolates made with different cocoa (Theobroma cacao L.) varieties. Brazilian Journal of Food and Techonology, Campinas, SP, v. 16, n. 3, p. 192-197, jul./set. 2013.

LOTICI, T.; SANTOS, E. F. D.; NOVELLO, D.; MANHANI, M. R.; SANCHES, F. L. F. Z. Adição de inulina em bolo de chocolate: composição físicoquímica e sensorial. Revista Brasileira de Inovação Tecnológica em Saúde, v. 3, n. 4, 2013.

GUIMARÃES, R. C. A. Propriedades químicas e térmicas de farinha desengordurada, concentrado e isolado proteico de Castanhas de baru 
(Dipteryx alata Vog.). 2009. Dissertação (Mestrado em Biotecnologia) - Universidade Católica Dom Bosco, Campo Grande, MS, 2009.

MOSCA, P. C.; OLIVEIRA, F. M; ZANARDO, F. D. C.; ROSATTO, F. M.; ALMEIDA, J. M.; PEREIRA, E. M. S.; TAVANO, O. L. Composição e aceitabilidade entre crianças de uma creche/escola de Uberaba-MG de bolo de chocolate adicionado de grãos de feijão cozidos. Revista Brasileira de Produtos Agroindustriais, Campina Grande, PB, v. 16, n. 4, p. 403-410, 2014.

MIQUELIM, J. N. Avaliação realógica e físico-química de bombons recheados com preparado de morango, laranja e maracujá em base açúcar fondant, gordura hidrogenada e chocolate branco. 2006. Dissertação (Mestrado em Tecnologia Bioquímico-Farmacêutica) - Universidade de São Paulo, São Paulo, 2006.

REIS, E. C. Análise físico-química e microbiológica de bombons artesanais. 2011. 36 f. Trabalho de Conclusão de Curso (Curso Superior de Tecnologia em Processos Químicos) - Universidade Tecnológica Federal do Paraná, Apucarana, PR, 2011.

RICHTER, M.; LANNES, S. C. D. S. Bombom para dietas especiais: avaliação química sensorial. Ciência e Tecnologia de Alimentos, Campinas, SP, v. 27, n. 1, p. 193-200, jan./mar. 2007.

ROBINSON, R. K.; PHILL, M. A. D. Microbiologia lactológica. Zaragoza: Acribia, 1987. p.18-32.

SANTOS, A. A. O.; SILVA, I. V. C. E.; SANTOS, J. P. A. D.; SANTANA, D. G.; ALMEIDA, M. L.; MARCELLINI, P. S. Elaboração de biscoitos de chocolate com substituição parcial da farinha de trigo por polvilho azedo e farinha de albedo de laranja. Ciência Rural, Santa Maria, RS, v. 41, n. 3, p. 531536, mar. 2011.

SOUSA, L. C. et al. Avaliação microbiológica, físico-química e das condições de fabricação de bombom de chocolate com recheio de frutas. Revista Alimentos e Nutrição, Araraquara, SP, v. 21, n. 2, p. 305-310, jun. 2010.

TEJADA, T. S.; DIAS, P. A.; CONCEIÇÃO, R. C. A. S.; TIMM, C. D. Pathogenic and deteriorative microorganisms in homemade milk chocolate. Revista do Instituto Adolfo Lutz, São Paulo, v. 71, n. 1, p. 178-181, 2012.

VANDERZANT, C.; SPLITTSTOESSER, F. Compendium of methods for the microbiological examination offoods. 3. ed. Washington, DC: APHA, 1992. VERA, R.; SOUZA, E. R. B. Baru. Revista Brasileira Fruticultura, Jaboticabal, SP, v. 31, n. 1, mar. 2009. 